\title{
About Chaotization Mechanisms of the Distributed Dynamical Systems Which are Close to Discrete
}

\author{
YU.P. BLIOKH *, M.G. LYUBARSKY and V.O. PODOBINSKY \\ Plasma Electronics Institute of National Science Center "KFTI", Kharkov, Ukraine
}

(Received 15 February 1997)

\begin{abstract}
The investigations of stochastization mechanisms of distributed dynamical systems (DDS) are developed not so complete as stochastization of dynamical systems with concentrated parameters (CDS). Therefore the corresponding DDS which is close (in one or other sense) to the CDS under consideration is used. Such substitution means some roughening of an initial problem. However, there are such important stochastization mechanisms understanding properties, which are connected with system "distributivity" on principle. In this paper the conception of a proximity to the CDS is introduced for one particular class of the DDS. It is shown that such kind of systems has two stochastization mechanisms, one of which is common to the DDS and the corresponding CDS. Another stochastization mechanism inherent in DDS disappears under transition from DDS to CDS.

Distributed dynamical system considered below is not abstract but describes the set of concrete physical devices, for example, microwave oscillators. It may be devices in which kinetic energy of an accelerated electron beam is transformed into electromagnetic radiation energy, or oscillators with delayed feedback.
\end{abstract}

Keywords: Distributed dynamical system, Chaotization, Mapping

\section{THE DISTRIBUTED DYNAMICAL} SYSTEMS CLOSE TO DISCRETE ONES

We shall act in the following way: from the wellknown CDS we pass to the DDS, admitting the reverse passage to the limit. As an initial CDS let us choose the point map:

$$
x_{n+1}=F\left(x_{n}\right),
$$

where $x$ is the state vector of a dynamical system.
If this vector for some concrete physical system is a continuous function of time, then such discrete map appears under passage to the "discrete time". It means that the system state at time $t$ depends only on its state in time $t-T$ :

$$
x(t+T)=F(x(t)) .
$$

It is obvious that Eqs. (1) and (2) are same, but formally the first of them describes the CDS and the second one the DDS. Really, the behaviour of

*Corresponding author. 
system (1) is known, if initial value $x_{0}$ is defined. For the dynamical system (2) all future behaviour is known if function $x(t)$ is defined on time interval $0<t<T$. Such "distributivity" means that system (2) consists of continuous set of the independent systems (1).

Let us pass now from the "formal" distributivity to the "real" one, considering the system, which state in time $t$ depends on the system behaviour on time interval $(t-T-\varepsilon, t-T)$ :

$$
x(t+T)=\hat{\mathcal{F}}_{\varepsilon}\{x(t)\}
$$

where $\hat{\mathcal{F}}_{\varepsilon}$ is some functional, which is defined on the interval $(t-T-\varepsilon, t-T)$. If $\varepsilon \rightarrow 0$ system (3) passes to system (2). The parameter $\varepsilon$ may be considered as a measure of systems (3) and (1) "proximity". The differences between systems (1)-(3) are presented schematically on Fig. 1.
The distributed dynamical system under consideration is close to the concentrated one if $\varepsilon \ll 1$. The aim of this work is to answer the following question: when passage to limit $\varepsilon \rightarrow 0$, from DDS (3) to CDS (2) (or, that is the same, to (1)) does not lead to the essential loss of system properties?

We will consider below only one particular class of systems described by functional map (3): state vector $x$ is the scalar complex-valued function and functional $\hat{\mathcal{F}}$ has the following special form:

$$
\hat{\mathcal{F}}_{\varepsilon}\{x(t)\}=F\left(\left|\hat{G}_{\varepsilon}\{x(t)\}\right|\right) \mathrm{e}^{i \arg \hat{G}_{\varepsilon}\{x(t)\}},
$$

where $F$ is a smooth complex-valued function and $\hat{G}_{\varepsilon}$ is a linear functional

$$
\hat{G}_{\varepsilon}\{x(t)\}=\int_{0}^{\infty} g(\tau) x(t-\tau) \mathrm{d} \tau .
$$
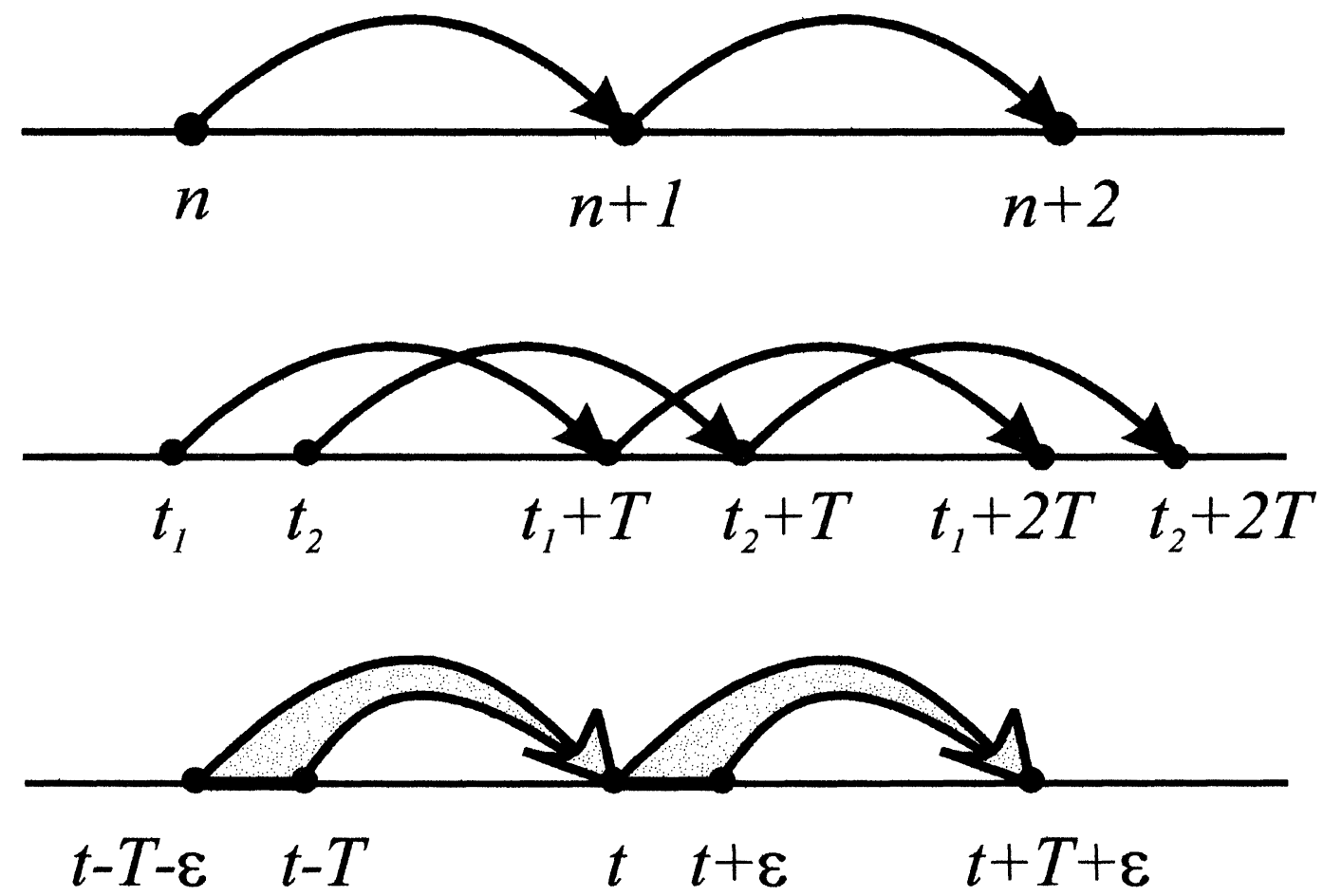

FIGURE 1 Schematical picture of number line transformations which are described by discrete dynamical system (1), "formally" distributed system with discrete time (2) and "real" distributed system (3). 
Here the core $g$ is negligibly small outside the interval $(0, \varepsilon)$.

So, the distributed dynamical system under consideration has the following special form:

$$
x(t+T)=F\left(\left|\hat{G}_{\varepsilon}\{x(t)\}\right|\right) \mathrm{e}^{i \arg \hat{G}_{\varepsilon}\{x(t)\}} .
$$

Although this is the simplest form of nonlinear functional in such sense that its "distributivity" is linear and "non-linearity" is scalar, this dynamical system describes the set of concrete microwave electronics devices with distributed wave-electron beam interaction (that is the authors' one of the main science interests). The details of such application are accounted in [14]. The initial boundary value problem that describes such devices operation is equivalent to functional equation (3) and the function $x$ is the output wave complex-valued amplitude. The special form (4) of functional $\hat{\mathcal{F}}$ is determined by additional properties of such kind of devices. The main of them are the phase shift invariance

$$
\hat{\mathcal{F}}\left\{\mathrm{e}^{i \varphi} x(t)\right\}=\mathrm{e}^{i \varphi} \hat{\mathcal{F}}\{x(t)\} \quad(0 \leq \varphi<2 \pi)
$$

and the causality principle.

Note that these properties are inherent in very broad class of dynamical systems, namely selfoscillating physical systems that admit the "fast time" averaging. In this case observation $x(t)$ is the high frequency signal envelope, and phase shift invariance follows from the system autonomy.

The radio engineering device known as "circular" generator, that is used in many experimental investigations of stochastization in the dynamical systems (see, for instance, [5,7]), submits one interesting example of dynamical system class under consideration. The circular generator represents closed circuit that consists of filter, non-linear amplifier and delay line connected in series. As $x$ the filter input signal complex-valued amplitude may be chosen. The filter transforms signal according to formula (5) where the core $g$ is determined by filter properties. Next the signal passes through non-linear amplifier that transforms it according to (4). Finally, after delay line the signal again arrives at filter input. The delay line output signal is the same as the filter input signal and this fact is expressed by formula (6).

Without loss of generality we may assume that

$$
\int_{0}^{\infty} g(\tau) \mathrm{d} \tau=1
$$

where $\tau=t / T$ is the dimensionless time. This condition may be fulfilled by proper norming of core $g$ and corresponding modification of function $F$. At the same time we suppose that $g$ is negligibly small outside the interval $(0, \varepsilon)$. DDS under consideration is close to discrete one, if $\varepsilon \ll 1$. If $\varepsilon=0$ then core $g$ is $\delta$-function (look at (7)) and dynamical system (6) turns itself into discrete system

$$
x(\tau+1)=F(|x(\tau)|) \mathrm{e}^{i \arg x(\tau)} .
$$

For investigation of these system stochastization mechanisms it is sufficient to watch only $x$ modulus, the evolution of which is determined by real dynamical system (1) with function

$$
\mathcal{F}=|F(\xi)|, \quad \xi \geq 0 .
$$

The argument does not play any role in this case because its change in every iteration is the function of current modulus value. As it will be shown below, this property of discrete systems distinguishes it strongly from DDS for which phase relations are very important.

Later on it is convenient to use Fourier transformation $\tilde{g}$ of core $g$ that describes the action of functional $\hat{G}$ on harmonic functions:

$$
\hat{G}\left\{\left(\mathrm{e}^{i \omega \tau}\right)\right\}=\tilde{g}(\omega) \mathrm{e}^{i \omega \tau} .
$$

Introduction of small parameter $\varepsilon$ means that every next derivative of function $\tilde{g}$ is smaller by an order than the previous one. If $\varepsilon=0$ then core $g$ is $\delta$-function and its Fourier transformation $\tilde{g}$ is equal to unity identically. 


\section{THE HARMONIC SOLUTION AND ITS STABILITY}

Fixed points of the map $\mathcal{F}$ and its instability condition play an important role in the theory of discrete systems. For DDS (6) the harmonic solutions play the role of fixed points due to functional $\hat{\mathcal{F}}$ phase shift invariance. In the approximation under consideration amplitude and frequency of any harmonic solution $x(\tau)=$ $a_{\omega} e^{i \omega \tau}, a_{\omega}>0$, must satisfy to equalities that follow from Eq. (6) and relation (10):

$$
\begin{aligned}
a_{\omega} & =\left|F\left(|\tilde{g}(\omega)| a_{\omega}\right)\right|, \\
\omega & =\arg F\left(|\tilde{g}(\omega)| a_{\omega}\right)+\arg \tilde{g}(\omega)+2 \pi k, \\
& k=0, \pm 1, \pm 2, \ldots
\end{aligned}
$$

If $\varepsilon=0$ then $\tilde{g}(\omega) \equiv 1$ and condition of harmonic solution existence is given by the first equation from system (11). This condition coincides with existence condition of a fixed point in the discrete mapping $\mathcal{F}$ which is determined by equality (9). In the case of non-zero but small $\varepsilon$ the system (11) may be solved by successive approximations. The frequencies of the harmonic solutions up to $\varepsilon$ are separated one from another by $2 \pi$ interval. Let us call them as fundamental frequencies of dynamical system (6).

Let us linearize Eq. (6) in the vicinity of harmonic solution in order to investigate its stability. It is possible for simplicity to put the frequency $\omega$ of the solution under consideration equal to zero.

As follows for disturbance $\delta x$ of harmonic solution $x(\tau)=a_{0}$ :

$$
\begin{aligned}
\delta x(\tau+1)= & {\left[|F|^{\prime}+|F|(\arg F)^{\prime}\right] \operatorname{Re} \hat{G}\{\delta x(\tau)\} } \\
& +i \operatorname{Im} \hat{G}\{\delta x(\tau)\},
\end{aligned}
$$

where all derivatives are calculated at the point $a_{0}$.

Due to right side non-analyticity it is necessary to find the solution $\delta x(\tau)$ of this equation in the form of superposition of two complex conjugate harmonics:

$$
\delta x(\tau)=C_{1} \mathrm{e}^{\nu \tau}+C_{2} \mathrm{e}^{\nu^{*} \tau} .
$$

As a result, the following characteristic equation is obtained:

$$
\begin{aligned}
e^{2 \nu} & -\frac{1}{2}\left\{\left[\tilde{g}(-\nu)+\tilde{g}^{*}(\nu)\right]\left(1+|F|^{\prime}\right)\right. \\
+ & {\left.\left[\tilde{g}(-\nu)-\tilde{g}^{*}(\nu)\right] a_{0}(\arg F)^{\prime}\right\} } \\
& +\tilde{g}(-\nu) \tilde{g}^{*}(\nu)|F|^{\prime}=0 .
\end{aligned}
$$

The assumption about the parameter $\varepsilon$ to be small is made in the main for analytical solving of this transcendental equation. The higher terms of the roots of this equation which were obtained as a series on $\varepsilon$ powers are shown below:

$$
\begin{aligned}
\nu_{k}^{(1)}= & \ln \|\left. F\right|^{\prime} \mid+i\left\{\frac{\pi}{2}\left[1-\operatorname{sign}\left(|F|^{\prime}\right)\right]+2 \pi k\right\}, \\
\nu_{k}^{(2)}= & 2(\pi k)^{2}\left[|\tilde{g}|^{\prime \prime}-(\arg \tilde{g})^{\prime} a_{0} \frac{(\arg F)^{\prime}}{1-|F|^{\prime}}\right] \\
& +i 2 \pi k \\
k= & 0, \pm 1, \pm 2, \ldots
\end{aligned}
$$

Here derivatives of function $\tilde{g}(\omega)$ are calculated at $\omega=0$ - the frequency of harmonic solution the stability of which is investigated.

The harmonic solution is unstable if at least one root has a positive real part. In spite of the fact that characteristic equation has infinite number of roots, their real parts have the same form for each sets (12) and (13). Therefore only two instability conditions exist:

$$
\begin{gathered}
\|\left. F\right|^{\prime} \mid>1, \\
|\tilde{g}|^{\prime \prime}-(\arg \tilde{g})^{\prime \prime} a_{0} \frac{(\arg F)^{\prime}}{1-|F|^{\prime}}>0 .
\end{gathered}
$$

Eigenvectors (the couple of coefficients $C_{1}$ and $C_{2}$ ) which show in what direction disturbance $\delta x$ evaluates are the same for these sets too. For set (12) the disturbance is real and only modulus of solution is changed on the initial stage of instability. For set (13) the disturbance is imaginary 
and only solution's phase is changed on initial stage of instability. It allows to call the instability mechanism that is connected with root set (12) as "amplitude" mechanism and another one, which is connected with root set (13), as "phase" mechanism.

At $\varepsilon=0, \tilde{g}(\omega) \equiv 1$ and the phase mechanism is absent because the roots (13) are imaginary. In this case instability may appear due to amplitude mechanism only, if the following condition is satisfied:

$$
\left|\mathcal{F}^{\prime}\right|>1,
$$

where function $\mathcal{F}$ is determined by (9). This condition is the same as the condition of fixed point of map $\mathcal{F}$ stability loss. It is so because the behaviour of dynamical system (6) if $\varepsilon=0$ is determined by discrete dynamical system (8) behaviour. If $\varepsilon$ is distinct from zero and system (6) becomes distributed then roots of set (13) may acquire the real part. In this case the harmonic solution can be unstable due to phase mechanism. For example, if $F \equiv 1$ then condition of amplitude instability is not fulfilled and stability loss may occur due to the second root set only. In this case condition (15) transforms into $|\tilde{g}|^{\prime \prime}>0$. For circular generator in this case was discovered in [7]. It means that harmonic solutions are unstable if the filter has a concave amplitude-frequency characteristic and the "perfect" stopping device is chosen as an amplifier.

If $\mathcal{F}^{\prime}<0$ (this case is considered in the Feigenbaum's theory) then amplitude and phase mechanisms are distinguished by frequencies that appear during instability development. In system (8) as soon as condition (16) is fulfilled, the mapping fixed point loses its stability, perturbation grows and oscillates with period 2. Analogously, if instability in system (6) appears due to amplitude mechanism (condition (14)), the growing perturbations have frequencies that are placed up to $\varepsilon$ at halfway between fundamental frequencies. It means that perturbation period is close to 2 . In case system (6) instability is connected with phase mechanism (condition (15)), the perturbations have frequencies that are close to fundamental. Such property allows to determine what kind of mechanisms is responsible for harmonic solution instability through numerical or experimental investigations of a system.

\section{DISTRIBUTED SYSTEM CHAOTIZATION AND SCENARIO OF TRANSITION TO CHAOS}

Results of this section are obtained by numerical modelling of dynamical system (6). For comparison of these results with Feigenbaum's theory let us assume that function $\mathcal{F}$ (it is connected with function $F$ by relation (9)) maps interval $[0,1]$ into itself and has one maximum and depends on parameter which changes system dynamics. Therefore we turn our attention to logistic map

$$
\mathcal{F}(\xi)=\lambda \xi(1-\xi), \quad 0 \leq \xi \leq 1,
$$

where parameter $\lambda$ is situated in the interval $(0,4)$.

For demonstration of two typical scenario of transit to chaos in the distributed system (6) let us choose logistic map as the function $F$ modulus and a linear function as $F$ argument:

$$
\arg F(\xi)= \pm \mu \xi,
$$

where $\mu>0$ is a parameter.

The core $g$ is chosen in such a way that stable harmonic solution exists when $\mu=0$. It means that the modulus of core Fourier transform must be convex function, according to condition (15).

If parameter $\mu$ is fixed, it is possible to observe the same scenario of transit to chaos as in discrete system (8). When parameter $\lambda$ is increased, then at first a stable harmonic solution arises. Further increasing of $\lambda$ leads to this solution stability loss, but new stable periodic solution appears. The period of solution up to $\varepsilon \ll 1$ is close to 2. This solution becomes unstable in its turn and further increasing of $\lambda$ leads to transit to 
chaos through infinite period doubling bifurcation chain just as in the corresponding discrete system. The bifurcation's magnitudes of $\lambda$ depend on core $g$ form. In other words, the distributed dynamical system under consideration behaves as the corresponding discrete system but its solutions are non-separable.

It is important to note the following. Let us represent the solution in the form

$$
x(\tau)=a(\tau) \mathrm{e}^{i(\omega \tau+\varphi(\tau))},
$$

where $\omega$ is the frequency of initial harmonic solution. It is characteristic for the scenario described above that phase $\varphi$ variation is small but amplitude $a$ variation is essential. It is natural to call this chaotization scenario (see condition (15)) as "amplitude" one.

The chaotization of solution happens by other way if parameter $\lambda$ is fixed but parameter $\mu$ increases. Let us choose the $\lambda$ value so that stable harmonic solution exists under $\mu=0$. If the sign in (17) is chosen so that inequality (15) may be valid, harmonic solution becomes unstable when $\mu$ exceeds the corresponding critical value. After harmonic solution collapse any bifurcations do not happen. The system already turns to alternating chaotic motion [6]. It means that solution on some intervals has regular form that slowly evolves with time and on other intervals the system's behaviour is chaotic. The characteristic modulation time scale is equal to 1 (time delay). When parameter $\mu$ increases then the part of chaotic motion grows and the regular one decreases up to complete disappearance. On the input stage of chaotization which is connected with condition (15), the amplitude $a(\tau)$ is practically constant and only phase $\varphi(\tau)$ varies. That is why this mechanism of transit to alternating chaos may be called as "phase" one.

The system (6) motion under phase chaotization mechanism may be represented as a random jump between two or more unstable harmonic solutions whose frequencies are the same as frequencies of the system. This behaviour is demonstrated on Fig. 2, where evolution of amplitude $a(\tau)$ and momentary frequency $\omega=\mathrm{d} \varphi / \mathrm{d} t$ are represented. The parameter $\lambda=1.2$ is chosen so small in order to avoid an amplitude instability mechanism. ${ }^{\dagger}$ The magnitude of $\mu$ is chosen slightly above its threshold value which corresponds to instability rise. The analogous dependencies $a(\tau)$ and $\omega(\tau)$, which are typical for amplitude instability mechanism, are represented on the Fig. 3 for comparison.

Solution spectra differ in accordance with chaotization mechanism. The spectrum that corresponds to amplitude chaotization mechanism is more compact and homogeneous (see Fig. 5). The spectrum with preferred fundamental frequencies and broad pedestal corresponds to the phase mechanism (see Fig. 4). This difference may be explained in such a way. Under amplitude chaotization mechanism every period doubling bifurcation leads to appearance of new frequencies between early existing. Under phase mechanism intermediate frequencies arise due to system non-linearity and fundamental frequencies non-equidistance only.

As an example of stochastic behaviour of a system under consideration, when both the instability mechanisms act simultaneously, the corresponding spectrum of solution is represented on Fig. 6.

If parameters $\lambda$ and $\mu$ are changed simultaneously or function $F$ is more complicated, or $\varepsilon$ is not so small then chaos transit scenario may be more complicated. For example in [1] the case is presented when after two period doubling bifurcations chaotization happens according to a scenario that is typical for phase mechanism.

\section{CONCLUSION}

In spite of the fact that phase space of the distributed dynamical system under consideration is

\footnotetext{
${ }^{\dagger}$ The harmonic solution stability loss and birth of periodic solution happens if $\lambda \simeq 3$.
} 


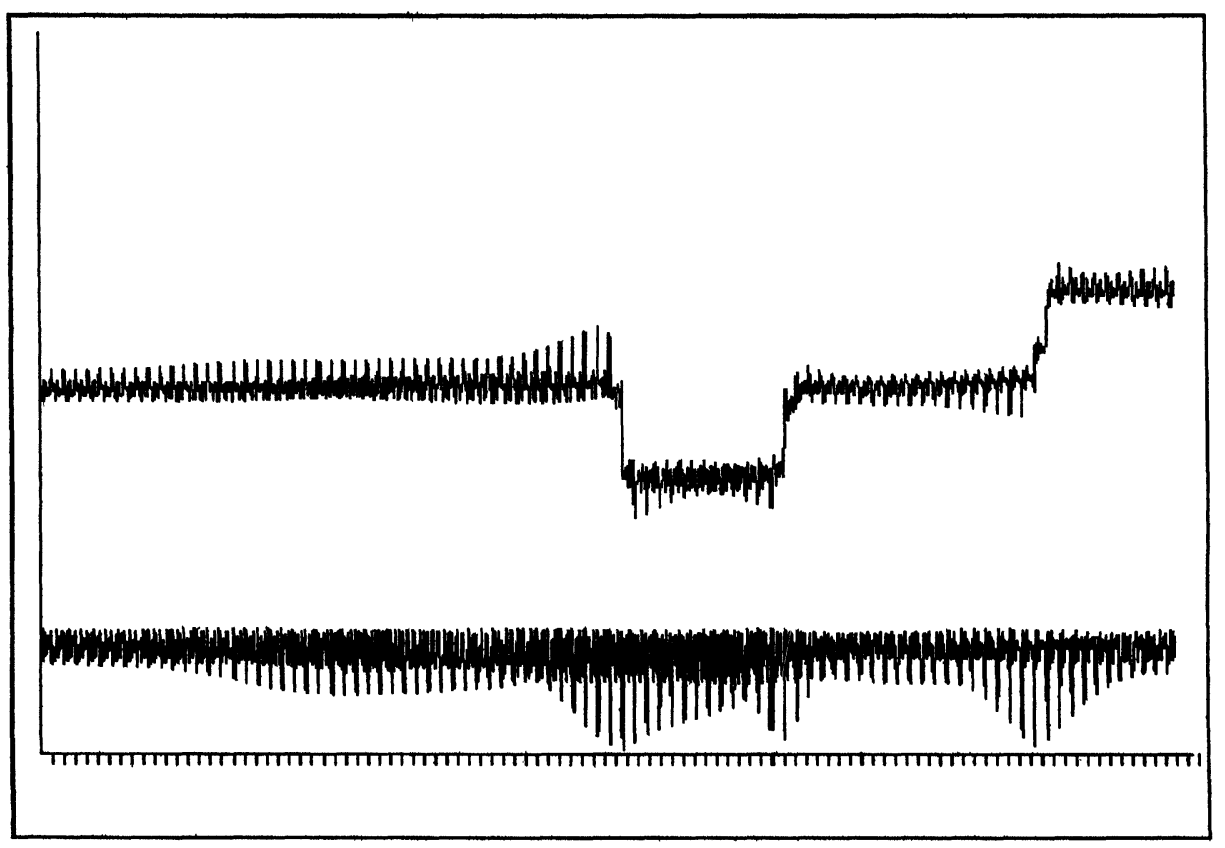

FIGURE 2 Time evolution of a momentary frequency $\omega(\tau)$ (above) and amplitude $a(\tau)$ (below) under phase instability mechanism.

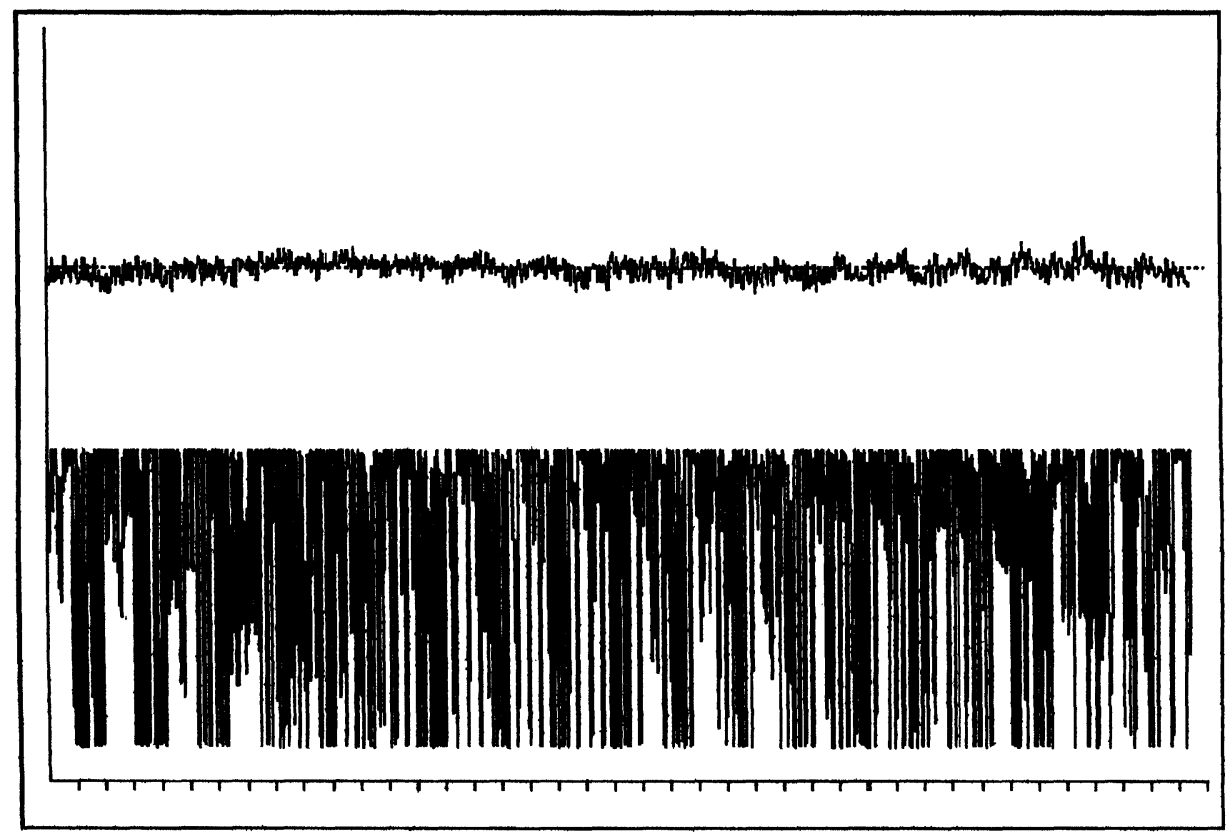

FIGURE 3 Time evolution of a momentary frequency $\omega(\tau)$ (above) and amplitude $a(\tau)$ (below) under amplitude instability mechanism. 


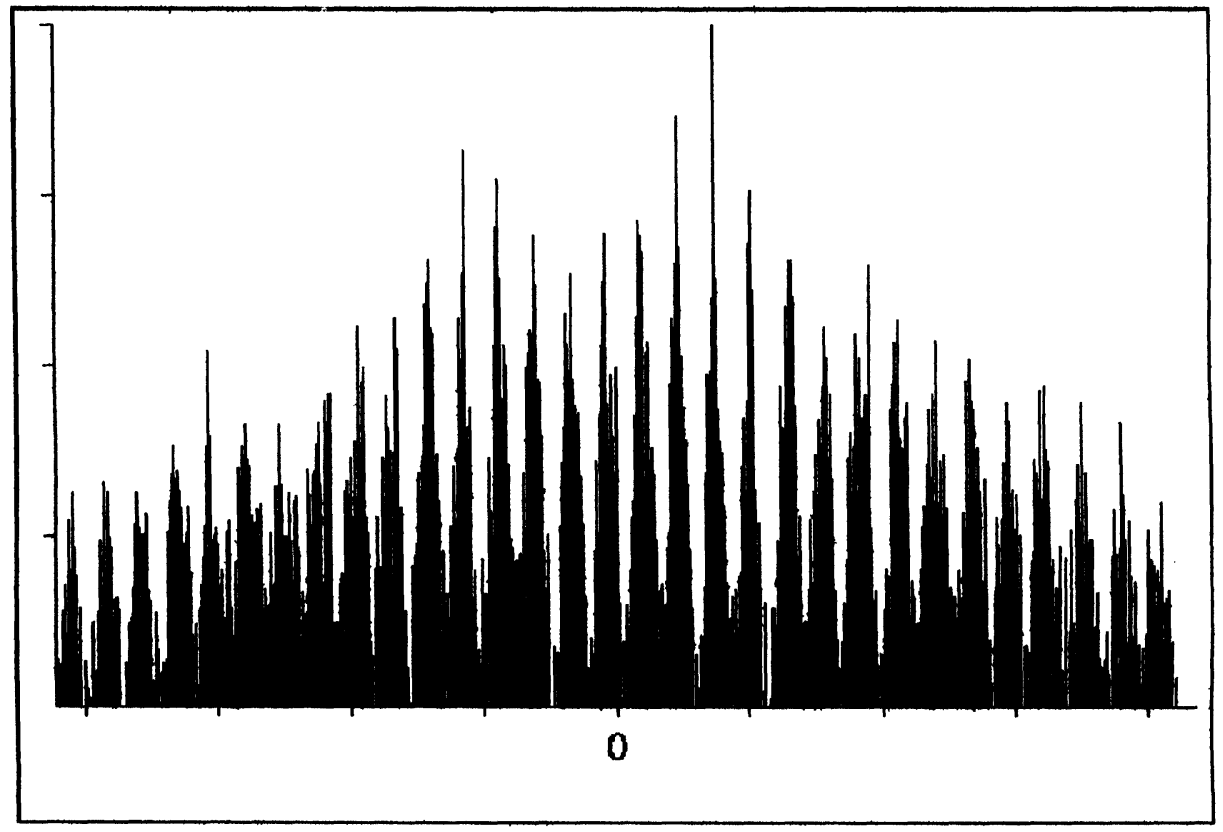

FIGURE 4 The solution spectrum under phase instability mechanism.

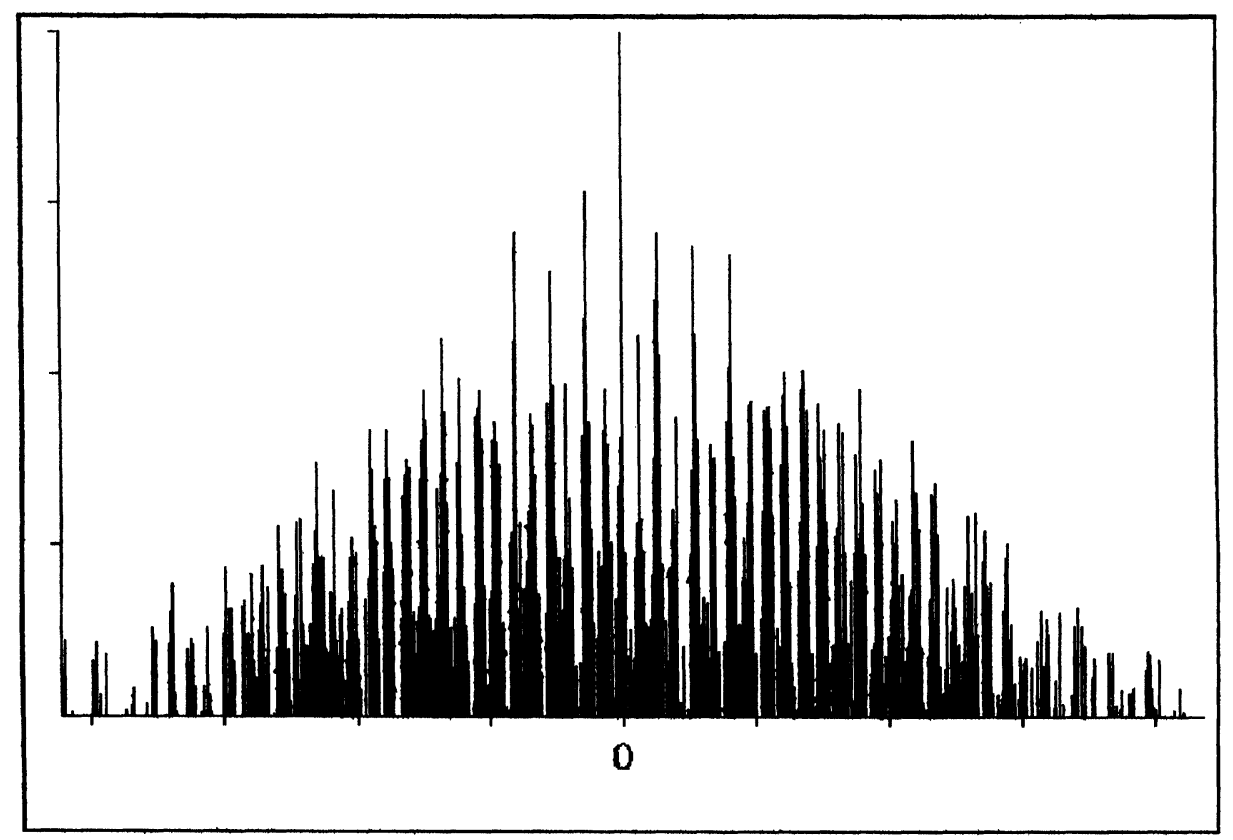

FIGURE 5 The solution spectrum under amplitude instability mechanism. 


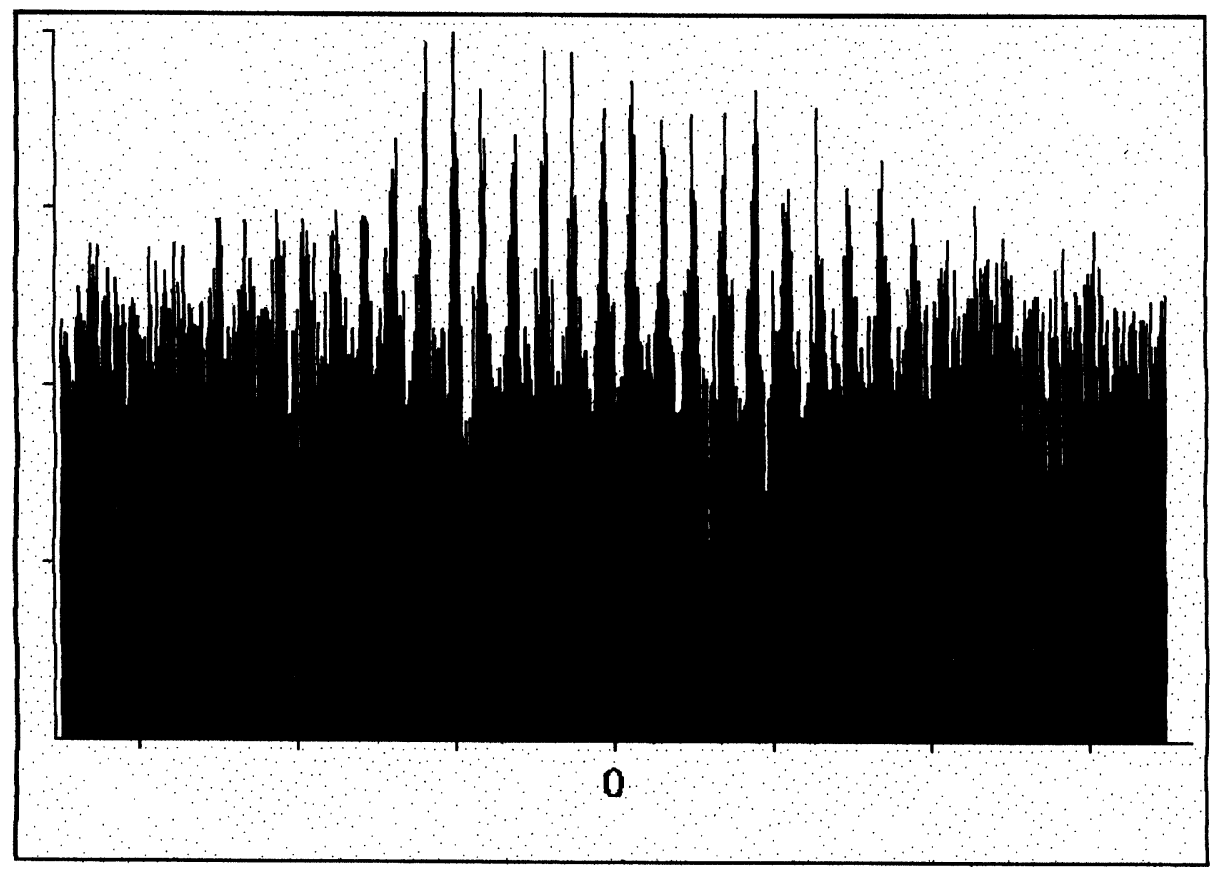

FIGURE 6 The solution spectrum when amplitude and phase mechanisms act simultaneously.

infinite, this system has only two main stochastization mechanisms. Corresponding chaos transit scenarios are well known: it is the sequence of period doubling bifurcations, or the hard transit to alternating chaos. The main properties of the chaotic regime (spectral density, correlation function, etc.) may be very different and depend strongly on chaotization mechanism. It is possible to realize any of these mechanisms by choice of system parameters and in this way to obtain the chaotic regime with required properties.

In this paper only the simplest example of a DDS is presented. During investigation and development of the concrete microwave devices we searched through various map functions $F$ and functionals $\hat{G}$. As general conclusions of these investigations it is possible to maintain the following. The narrow and homogeneous solution spectrum rises if stochastization is caused due to the amplitude mechanism, the phase mechanism must be absent. The broad but strongly inhomogeneous spectrum appears only if chaotization is connected with phase mechanism. There are some methods of spectrum "smoothing" (the experimental examples of such homogeneous spectrum are presented in $[2,4])$, but these methods' discussion deviates from the framework of this paper.

\section{References}

[1] Bliokh, Yu.P., Borodkin, A.V., Fainberg, Ya.B. et al., Izv. VUZov, Ser. Applied Nonlinear Dynamics, 1992, Vol. 1, No. 1-2, pp. 34-49.

[2] Bliokh, Yu.P., Fainberg, Ya.B., Lyubarskii, M.G. et al., Pl. Phys. Reports, 1994, Vol. 20, No. 8, pp. 675-678.

[3] Bliokh, Yu.P., Fainberg, Ya.B., Lyubarskii, M.G. and Podobinskii, V.O., Chaos, Solitons and Fractals, UK, 1996, Vol. 7, No. 2, pp. 273-292.

[4] Bliokh, Yu.P., Fainberg, Ya.B., Kornilov, E.A. et al., 10th Int. Conf. on High Power Particles Beams, 1994, San Diego, CA, Vol. 2, pp. 953-957.

[5] Kuznetsov, S.P., Izv. VUZov, Ser. Radiophysics, 1982, Vol. 25, No. 12, pp. 1410-1428.

[6] Feigenbaum, M.J., Comm. Math. Phys., 1980, Vol. 77, p. 65.

[7] Gonorovskii, J.S., Radiotechnika, 1958, Vol. 13, No. 5, pp. $19-30$. 


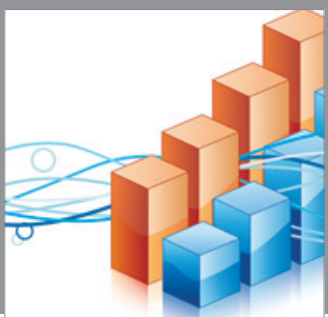

Advances in

Operations Research

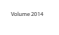

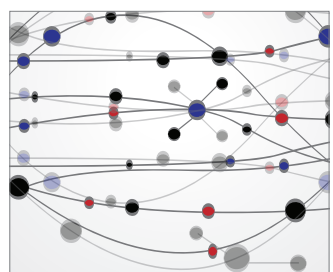

\section{The Scientific} World Journal
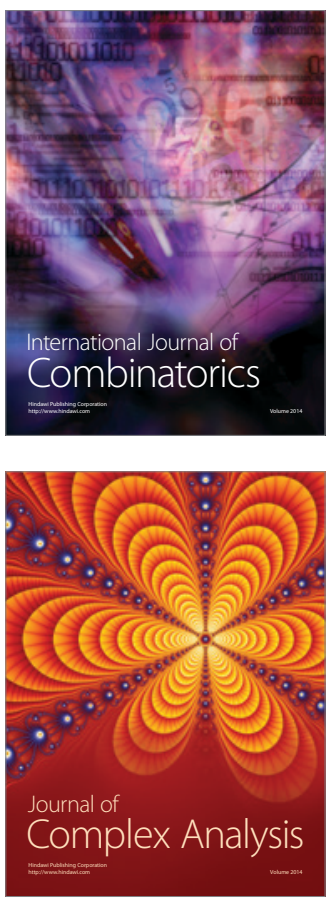

International Journal of

Mathematics and

Mathematical

Sciences
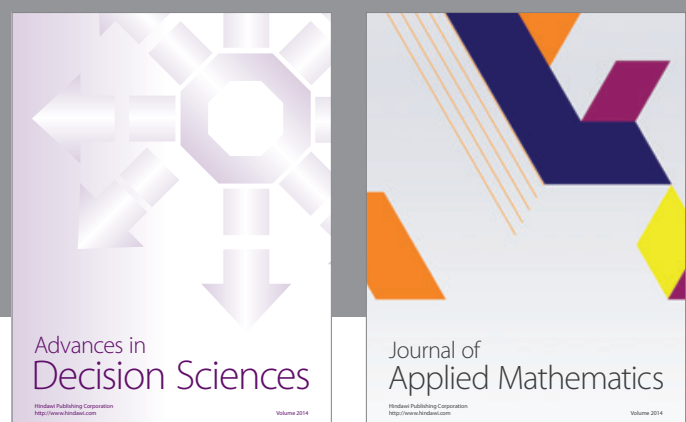

Journal of

Applied Mathematics
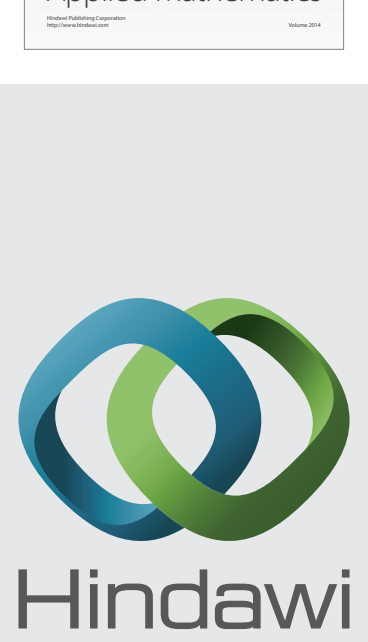

Submit your manuscripts at http://www.hindawi.com
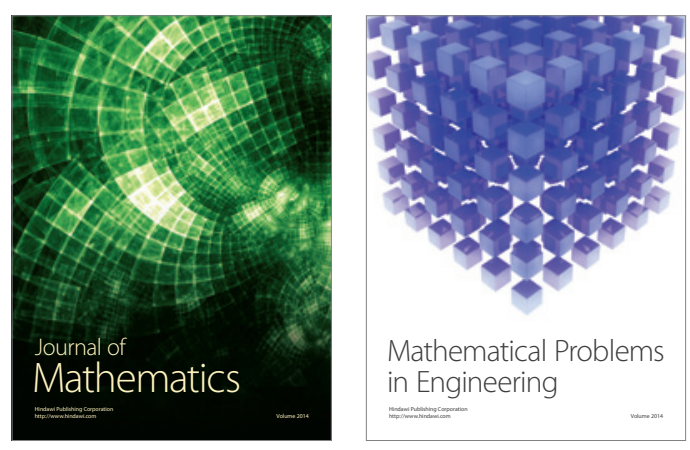

Mathematical Problems in Engineering
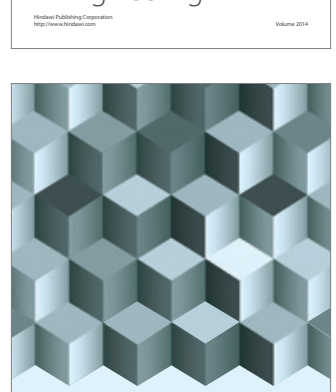

Journal of

Function Spaces
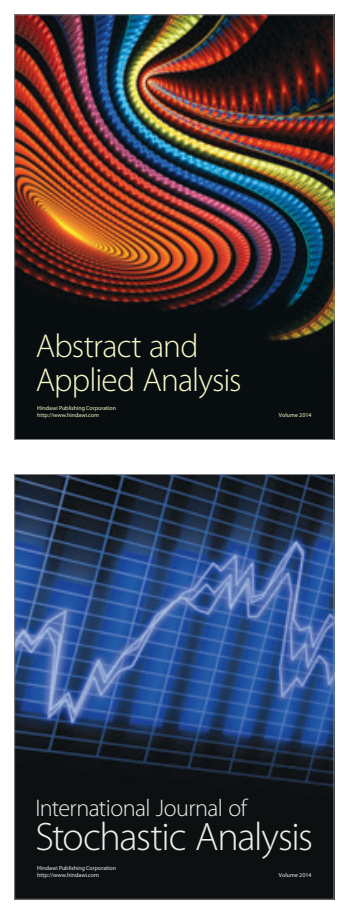

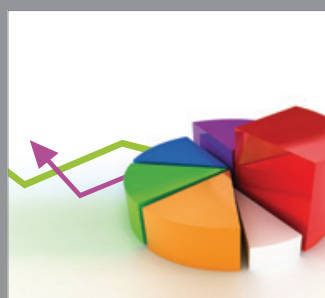

ournal of

Probability and Statistics

Promensencen
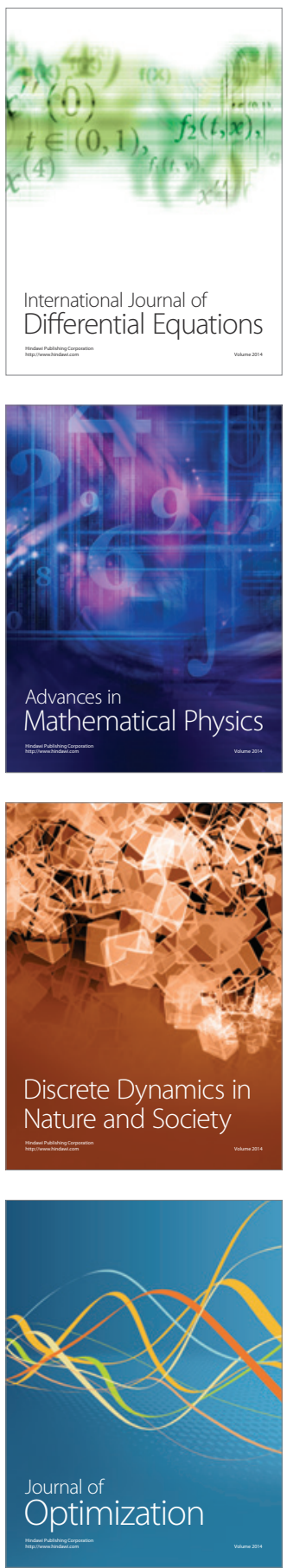